\title{
Visual Analysis of Biomarkers Reveals Differences in Lipid Profiles and Liver Enzymes before and after Gastric Sleeve and Bypass
}

\author{
Marijn Marthe Georgine van Berckel ${ }^{a}$ Saskia L.M. van Loon ${ }^{b, c} \quad$ Arjen-Kars Boer $^{b}$ \\ Volkher Scharnhorst ${ }^{\mathrm{b}, \mathrm{c}}$ Simon W. Nienhuijs ${ }^{\mathrm{a}}$ \\ ${ }^{a}$ Department of Surgery, Catharina Hospital, Eindhoven, The Netherlands; ${ }^{b}$ Department of Clinical Chemistry, \\ Catharina Hospital, Eindhoven, The Netherlands; ${ }^{C}$ Department of Biomedical Engineering, Eindhoven University of \\ Technology, Eindhoven, The Netherlands
}

\section{Keywords}

Bariatric surgery · Sleeve gastrectomy · Gastric bypass .

Biomarker · Beanplot

\begin{abstract}
Introduction: Bariatric surgery results in both intentional and unintentional metabolic changes. In a high-volume bariatric center, extensive laboratory panels are used to monitor these changes pre- and postoperatively. Consecutive measurements of relevant biochemical markers allow exploration of the health state of bariatric patients and comparison of different patient groups. Objective: The objective of this study is to compare biomarker distributions over time between 2 common bariatric procedures, i.e., sleeve gastrectomy (SG) and gastric bypass (RYGB), using visual analytics. Methods: Both pre- and postsurgical (6, 12, and 24 months) data of all patients who underwent primary bariatric surgery were collected retrospectively. The distribution and evolution of different biochemical markers were compared before and after surgery using asymmetric beanplots in order to evaluate the effect of primary SG and RYGB. A beanplot is an alternative to the boxplot that allows an easy and thorough
\end{abstract}

\begin{tabular}{ll}
\hline karger@karger.com & $\begin{array}{l}\text { (C) } 2021 \text { The Author(s) } \\
\text { Published by S. Karger AG, Basel }\end{array}$ \\
www.karger.com/ofa & This article is licensed under the Creative Commons Attribution- \\
Karger & $\begin{array}{l}\text { NonCommercial-NoDerivatives 4.0 International License (CC BY- } \\
\text { NC-ND) (http://www.karger.com/Services/OpenAccessLicense). } \\
\text { Usage and distribution for commercial purposes as well as any dis- } \\
\text { tribution of modified material requires written permission. }\end{array}$ \\
BOPEN ACCESS &
\end{tabular}

visual comparison of univariate data. Results: In total, 1,237 patients (659 SG and 578 RYGB) were included. The sleeve and bypass groups were comparable in terms of age and the prevalence of comorbidities. The mean presurgical BMI and the percentage of males were higher in the sleeve group. The effect of surgery on lowering of glycated hemoglobin was similar for both surgery types. After RYGB surgery, the decrease in the cholesterol concentration was larger than after SG. The enzymatic activity of aspartate aminotransferase, alanine aminotransferase, and alkaline phosphate in sleeve patients was higher presurgically but lower postsurgically compared to bypass values. Conclusions: Beanplots allow intuitive visualization of population distributions. Analysis of this large population-based data set using beanplots suggests comparable efficacies of both types of surgery in reducing diabetes. RYGB surgery reduced dyslipidemia more effectively than SG. The trend toward a larger decrease in liver enzyme activities following SG is a subject for further investigation.

(c) 2021 The Author(s)

Published by S. Karger AG, Basel

M.M.G.B. and S.L.M.L. contributed equally to this work.
Marijn Marthe Georgine van Berckel

Department of Surgery, Catharina Hospital Michelangelolaan 2

NL-5623 EJ Eindhoven (The Netherlands) marijnvanberckel@gmail.com 


\section{Introduction}

The only effective long-lasting treatment for morbid obesity is bariatric surgery $[1,2]$. Both in terms of weight loss and in relation to improvement of comorbidities and reduction of subsequent mortality, bariatric surgery is the treatment of choice $[3,4]$. These beneficial interventions are, however, also associated with complications or expected consequences, e.g., micronutrient deficiencies. These should be taken into account when indicating operative procedures [5]. Consequently, European and American guidelines advise routine metabolic and nutritional monitoring after all bariatric surgical procedures at specified, periodic follow-up time points [6, 7]. However, despite all of the recommendations, the 2017 guideline on micronutrients of the American Society for Metabolic and Bariatric Surgery showed an increase in micronutrient deficiencies after weight loss surgery [5].

Sleeve gastrectomy (SG) and gastric bypass (RYGB) are the most commonly performed bariatric surgical procedures. Both have been proven to be effective for the treatment of morbid obesity. RYGB is a restrictive procedure with a malabsorptive component, and it promotes weight loss and metabolic improvement also through additional hormone-mediated mechanisms. SG is a restrictive technique, with possibly beneficial effects on a variety of obesity-related hormones $[8,9]$. Recently, SG has overtaken RYGB as the most commonly performed type of bariatric surgery worldwide. From 2015 to 2018, SG accounted for $58.6 \%(n=305,242)$ of all bariatric procedures, while RYGB accounted for $31.2 \%(n=162,613)$ [10].

The research on the difference in metabolic and nutritional statuses after SG and RYGB is limited. In 2014, a meta-analysis on micronutrient deficiencies after bariatric surgery was published by Kwon et al. [11]; it included 9 small studies providing data on a total of 1,104 participants. The results were difficult to interpret due to inconsistency in definitions of deficiencies, as well as significant differences in follow-up times between the studies, in combination with a lack of preoperative information on deficiencies. Also, metabolic status is hard to compare between patients. The metabolic syndrome is reflected by comorbidities like type 2 diabetes mellitus (T2DM), hypertension, and dyslipidemia, which are interdependent. Metabolic monitoring should take into account parameters relevant for these comorbidities. Therefore, in our high-volume bariatric center extensive laboratory panels have been used to monitor patients pre- and postoperatively. As a result of using this approach since 2011, a comprehensive data set of biomarkers is available. These parameters allow exploration of the health state of bariatric patients and comparison of different patient groups.

The objective of this study is to compare biomarker distributions over time in patients who have undergone primary bariatric surgery. The focus lies on differences between SG and RYGB. To enable analytical interpretation of such large, complex data sets, visual analytics are used in the form of beanplots as an alternative to the boxplot [12-15]. This allows easy visual comparison of the univariate laboratory data between both types of surgery. Also, anomalies in the data such as bimodal distributions are easily spotted. Hereby, human and machine analyses are closely coupled, improving interpretability and enabling the discovery of new information.

\section{Methods}

Comprehensive laboratory data were collected from patients who underwent primary SG or RYGB surgery between January 2011 and July 2015 at the Catharina Hospital Obesity Center (Eindhoven, The Netherlands). Dutch guidelines, i.e., an optional preconditioning program, a shared decision making process for choosing between sleeve or bypass, and a mandatory follow-up program, were followed for screening. Patients who succeeded in the regular follow-up program, including laboratory measurements at least preoperatively and additionally 6, 12, and 24 months after surgery were included.

As a rule, patients with deficiencies, encountered in the preoperative laboratory measurement results, were prescribed supplements. Patients were advised to start using multivitamins around 6 weeks after surgery. Dieticians educated patients in selecting the optimal products after SG or RYGB. In case of a deficiency postoperatively, most frequently the treating physician prescribed supplements; in other cases this was done by a general practitioner.

Gender, age, BMI, date of surgery, and type of surgery were extracted from the electronic medical records. In addition, presence or absence of comorbidities (i.e., T2DM, hypertension, and dyslipidemia) before and after surgery was extracted from the medical records, according to local practice (see online suppl. material for criteria; see www.karger.com/doi/10.1159/000510401 for all online suppl. material) [16].

\section{Laboratory Data}

The bariatric laboratory panel included measurements of biomarkers reflecting hematological status, kidney function, liver function, thyroid function, glucose metabolism, lipid profile, and inflammation parameters. The biomarkers were analyzed on routine (immuno-) chemistry analyzers both before and after surgery during multiple outpatient follow-up visits. The included laboratory variables presented in the visual analysis were glycated hemoglobin (HbAlc; Tosoh G8, Sysmex), calcium, parathyroid hormone (PTH), triglycerides, cholesterol (total), high-density lipoprotein (HDL) cholesterol, aspartate aminotransferase (AST), alanine aminotransferase (ALT), and alkaline phosphatase (ALP; all Cobas, Roche Dx). An additional, calculated parameter was the cholesterol/HDL ratio. van Berckel et al. 
Table 1. Baseline characteristics

\begin{tabular}{lccc}
\hline Characteristic & SG $(n=659)$ & RYGB $(n=578)$ & $p$ value \\
\hline Female & 75 & 88 & $<0.001$ \\
Age at the time of surgery, years & $43.5 \pm 11.1$ & $43.0 \pm 9.9$ & 0.76 \\
BMI & $44.9 \pm 6.2$ & $42.2 \pm 4.1$ & $<0.001$ \\
T2DM & 19 & 18 & 0.72 \\
Hypertension & 40 & 34 & 0.04 \\
Dyslipidemia & 23 & 18 & 0.07 \\
\hline
\end{tabular}

Values are presented as percents or mean \pm SD. The presence of comorbidities (T2DM, hypertension, and dyslipidemia) was extracted from medical records, according to local practice (see online suppl. material for criteria) [16].

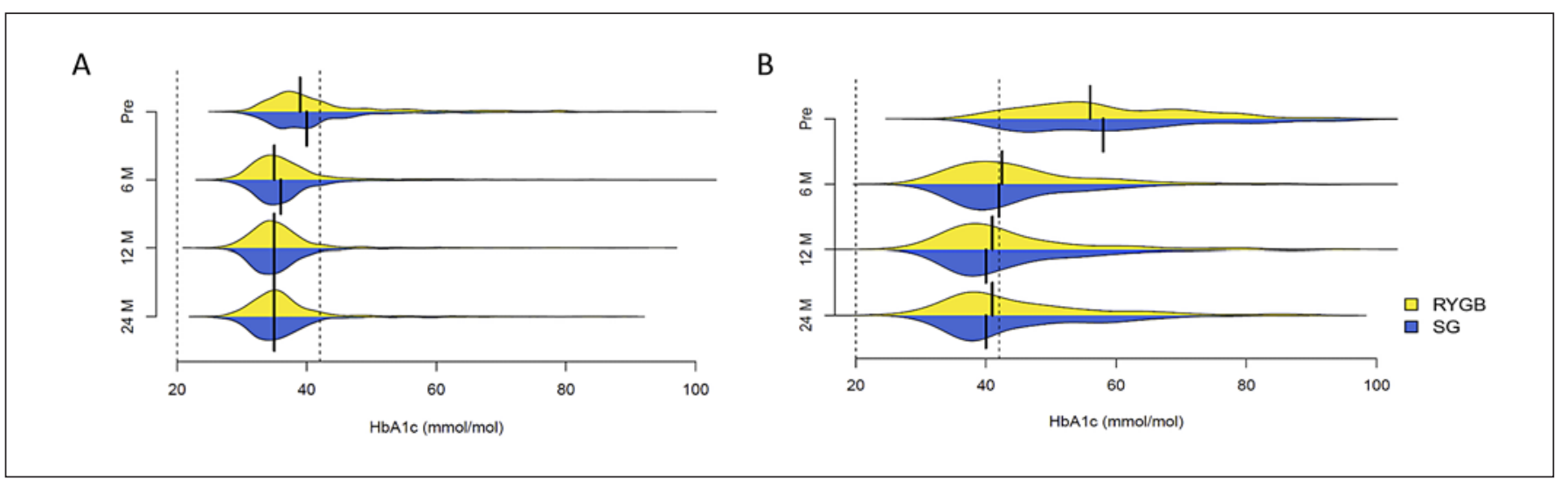

Fig. 1. Beanplots of the biomarker HbAlc showing the distribution of patients with either RYGB (top) or SG (bottom) and their evolution over time. The distributions are plotted for the total population $(n=1,237)(\mathbf{A})$ and for the subpopulation of patients with T2DM $(n=233)$ (B). The solid vertical lines within each distribu-

The laboratory data were merged with the extracted patient data. For each patient and for each time period, the data pair with the smallest number of days between them was chosen as a record for visualization. The presurgical period was defined as the period of 1 year before surgery. Follow-up periods were defined in time slots of 6 months, containing 3 months before and after the scheduled follow-up moment.

\section{Statistical Analysis}

Patient characteristics are listed as means $( \pm \mathrm{SD})$ or medians (IQR), depending on their distribution. Continuous data were compared with the Wilcoxon rank sum test for nonparametric data. Categorical data were compared using the Fisher exact test. $p<0.05$ was considered statistically significant.

Visual comparison between SG and RYGB was performed using beanplots. The density shape used was a polygon given by a normal density trace using the beanplot package in $\mathrm{R}$ [12]. In the beanplot, the estimated density of the distributions was given, including the median. By using asymmetric beanplots, each surgery type has one side of the so called "bean." For different laboratory parameters, their distribution and evolution before and after surgery were compared. The Wilcoxon rank sum test with continuity

Visual Analysis of Biomarkers after

Gastric Sleeve and Bypass tion indicate the median of each distribution. The dashed vertical lines within each plot indicate the reference values for $\mathrm{HbA1c}$, i.e., $20-42 \mathrm{mmol} / \mathrm{mol}$. Pre, presurgery; $6 \mathrm{M}, 12 \mathrm{M}$, and $24 \mathrm{M}$, respectively, 6, 12, and 24 months after surgery.

correction was used to compare medians between both groups at different time points. $p<0.05$ was considered statistically significant.

Statistical software $\mathrm{R}$ version 3.6.0 with the rms package by Harrell Jr. was used for data analysis and model building.

\section{Results}

The results of 659 SG and 578 RYGB surgeries were included in the analysis. Baseline characteristics are reported in Table 1. The mean presurgical BMI, the percentage of males, and the percentage of patients with hypertension were significantly higher in the sleeve group.

\section{Glucose Metabolism (Hb1Ac)}

In a comparison between RYGB and SG, the effect of surgery on lowering of $\mathrm{HbAlc}$ was similar for both surgery types (Fig. 1). After a significant decrease in the first 
Table 2. Biomarker comparison between RYGB and SG

\begin{tabular}{|c|c|c|c|c|c|c|c|}
\hline Calcium, mmol/L & $2.1-2.55$ & 2.37 & 2.37 & 0.48 & 2.32 & 2.35 & $<0.001$ \\
\hline $\mathrm{PTH}, \mathrm{pmol} / \mathrm{L}$ & $1.6-6.9$ & 6.4 & 6.8 & $<0.01$ & 5.9 & 5.4 & $<0.001$ \\
\hline Cholesterol-HDL ratio & $<5$ & 4.3 & 4.3 & 0.83 & 2.7 & 3.1 & $<0.001$ \\
\hline AST, U/L & $<30^{\mathrm{a}}$ & 23 & 24 & 0.04 & 22 & 20 & $<0.001$ \\
\hline ALT, U/L & $<35^{\mathrm{a}}$ & 26 & 28 & 0.03 & 22 & 19 & $<0.001$ \\
\hline ALP, IU/L & $40-120$ & 82 & 80 & 0.78 & 86 & 72 & $<0.001$ \\
\hline
\end{tabular}

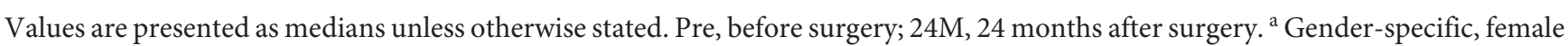
values were chosen as the majority of the population is female.

Table 3. Comparison of HbA1c levels between patients with T2DM and patients without T2DM

\begin{tabular}{|c|c|c|c|c|c|c|}
\hline $\mathrm{HbAlc} \mathrm{c}^{\mathrm{a}}, \mathrm{mmol} / \mathrm{mol}$ & \multicolumn{3}{|l|}{ Pre } & \multicolumn{3}{|l|}{$24 \mathrm{M}$} \\
\hline Without T2DM $(n=1,004)$ & $38(36-41)$ & $39(36-41)$ & 0.06 & $35(33-37)$ & $34(32-37)$ & 0.31 \\
\hline
\end{tabular}

Values are presented as medians (25th-75th percentile). Pre; before surgery; 24M, 24 months after surgery. ${ }^{\text {a }}$ Reference interval: 20-42 $\mathrm{mmol} / \mathrm{mol}$.

6 months for both types of surgery, the levels of Hb1Ac stabilized afterwards. No difference was seen in the levels of $\mathrm{Hb} 1 \mathrm{Ac}$ between RYGB and SG after 2 years (i.e., median [25th-75th percentile]: 35 [33-38] vs. 35 [33-38] $\mathrm{mmol} / \mathrm{mol} ; p=0.68$; Table 2). The distribution width of $\mathrm{HbA1c}$ values also decreased after surgery; however, extreme values $(>80 \mathrm{mmol} / \mathrm{mol})$ remained after surgery (Fig. 1a). Based on the right-hand-sided tail of the beans, SG has a longer tail compared to RYGB. Nonetheless, after surgery the distributions normalized to the wellknown bell-shape, where the majority has HbAlc-values within the normal range. In a comparison between RYGB and SG, the effect of surgery on lowering HbAlc was similar for both surgery types.

As HbA1c is related to T2DM and only about one fifth of our population has T2DM (Table 1 ), a subanalysis was done within this specific patient group $(n=233)$. The median $\mathrm{HbA} 1 \mathrm{c}$ level was higher in the subgroup with T2DM compared to patients without T2DM (Table 3). However, a comparison of HbA1c levels between RYGB and SG showed no significant differences either before or after surgery. Plotting of the distribution of $\mathrm{HbAlc}$ levels of this subgroup over time shows a prominent shift towards the normal range (Fig. 1b). The shape and width of the distributions indicate possible subgroups within this patient group that also remain after surgery.

\section{Lipid Profile (Triglycerides, Total Cholesterol, and Cholesterol-HDL Ratio)}

In SG and RYGB patients a major decline in triglyceride concentrations was seen 6 months postoperatively. During further follow-up, a gradual decline was seen. After 24 months, RYGB patients reached a significant lower triglyceride concentration compared to SG patients (i.e., 1.0 [0.74-1.3] compared to $1.1[0.8-1.6] \mathrm{mmol} / \mathrm{L} ; p<$ 0.001 ; Table 2). Before surgery, the shape of the beanplot revealed multiple subgroups within each surgery group, i.e., a group with triglyceride concentrations below the target value of $2 \mathrm{mmol} / \mathrm{L}$ and a smaller group with concentrations $>2 \mathrm{mmol} / \mathrm{L}$. This feature disappeared in the 
Fig. 2. Beanplots of cholesterol. Beanplots of the biomarkers triglycerides (A), total cholesterol (B), and HDL-cholesterol ratio (C) showing the distribution of patients with either RYGB or SG and their evolution over time. The distributions are plotted for the total population $(n=1,237)$. The solid vertical lines within each distribution indicate the median of each distribution. The dashed vertical lines within each plot indicate the specific reference or target values. Pre, presurgery; $6 \mathrm{M}, 12 \mathrm{M}$, and $24 \mathrm{M}$, respectively, 6,12 , and 24 months after surgery.

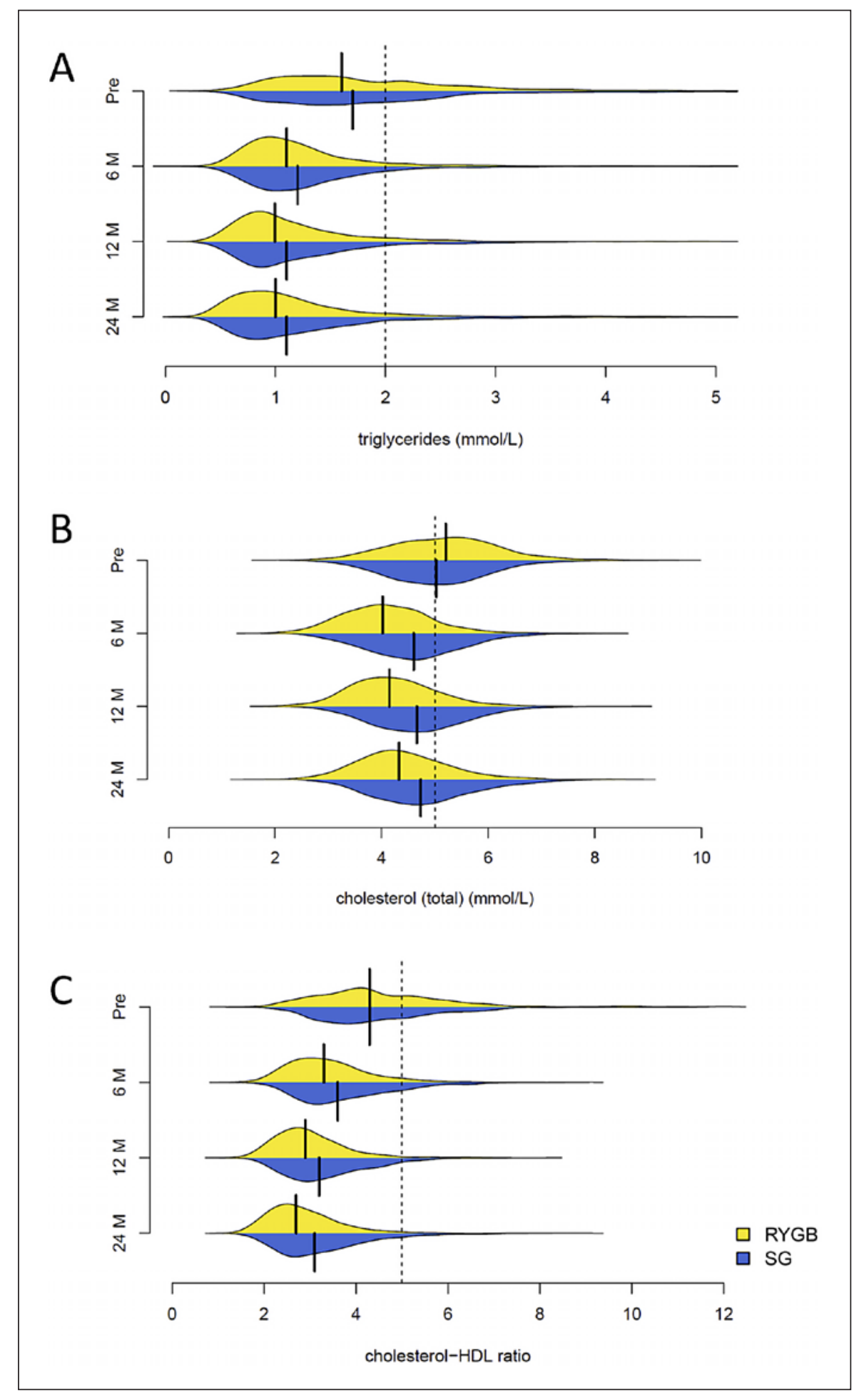

follow-up period, where the beanplot had the shape of a skewed normal distribution (Fig. 2a). After surgery a proportion of the population in both groups continued to have triglyceride concentrations above the target value, but this proportion was significantly smaller compared to before surgery.

A higher preoperative total cholesterol concentration was seen in RYGB patients compared to SG patients (Ta- 
Fig. 3. Beanplots of liver enzymes. Beanplots of the biomarkers AST (A), ALT (B), and ALP (C) showing the distribution of patients with either RYGB or SG and their evolution over time. The distributions are plotted for the total population $(n=1,237)$. The solid vertical lines within each distribution indicate the median of each distribution. The dashed vertical lines within each plot indicate the specific reference values. Pre, presurgery; $6 \mathrm{M}, 12 \mathrm{M}$, and $24 \mathrm{M}$, respectively, 6,12 , and 24 months after surgery.

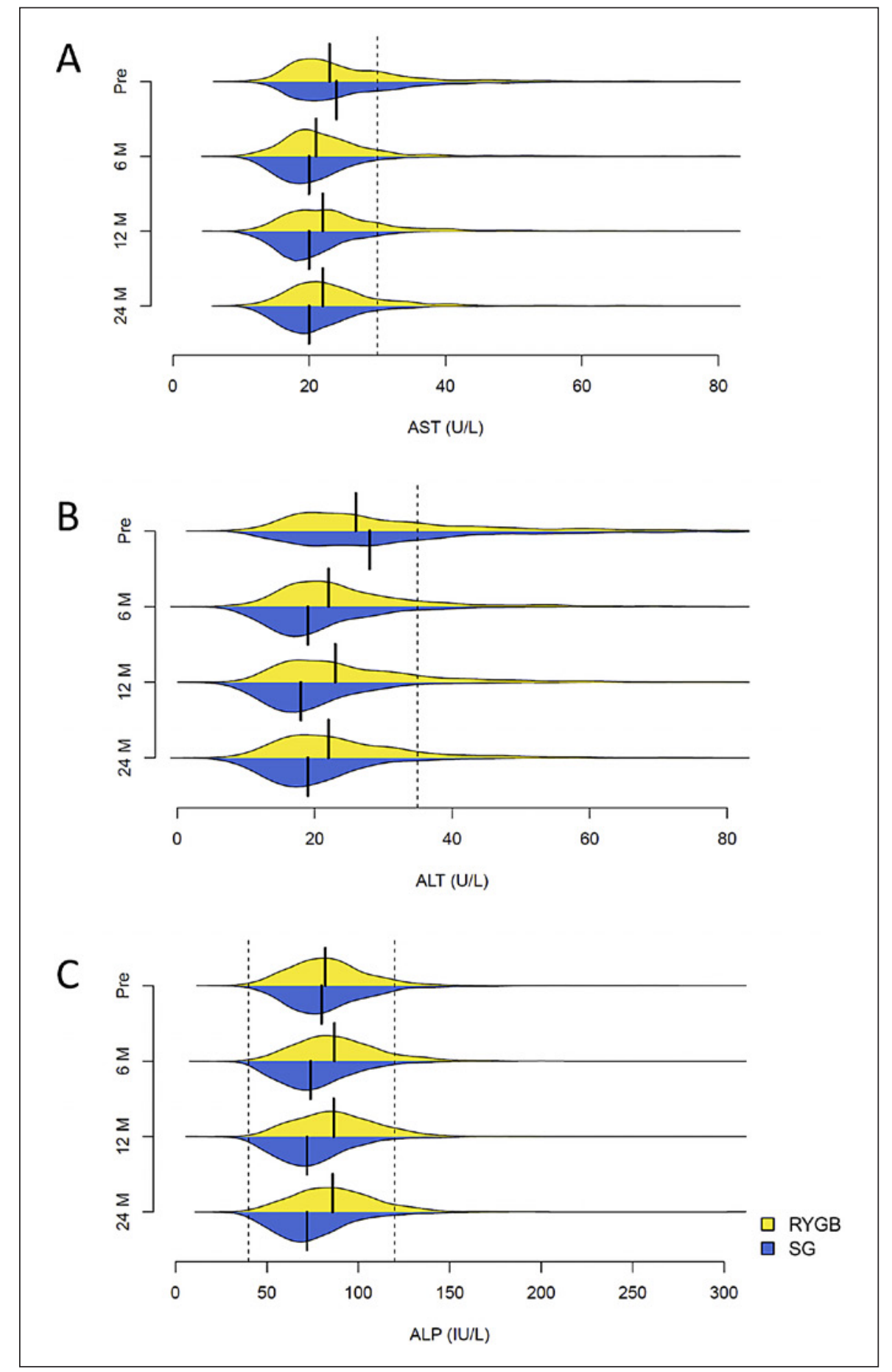

ble 2). Six months after surgery, a major decline was observed in RYGB patients. Thereafter, the levels of cholesterol consistently increased in small steps (Fig. 2b). In SG patients, the same distribution over time was encountered but to a lesser extent. This resulted in a significantly lower median level of cholesterol 24 months after
RYGB, i.e., 4.3 (3.9-4.9) $\mathrm{mmol} / \mathrm{L}$ compared to 4.7 (4.25.4) $\mathrm{mmol} / \mathrm{L}$ in SG patients $(p<0.001)$. The beanplot clearly shows the shift of the majority of patients having cholesterol concentrations above the target value of $5 \mathrm{mmol} / \mathrm{L}$ before surgery towards below $5 \mathrm{mmol} / \mathrm{L}$ after surgery (Fig. 2b). 
A

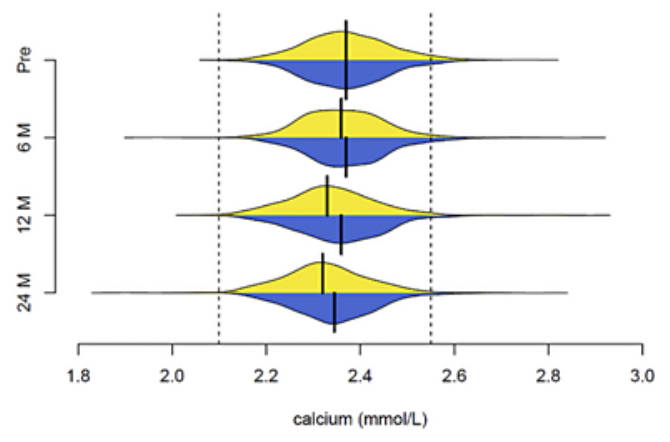

B

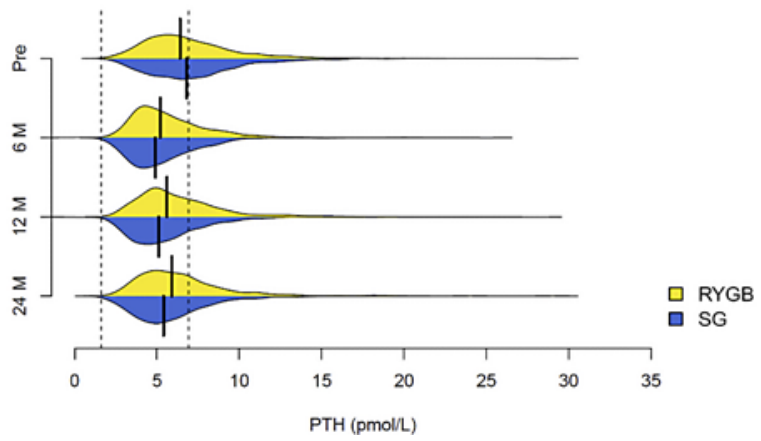

Fig. 4. Beanplots of calcium regulation. Beanplots of the biomarkers calcium (A) and PTH (B) showing the distribution of patients with either RYGB or SG and their evolution over time. The distributions are plotted for the total population $(n=1,237)$. The solid vertical lines within each distribution indicate the median of each distribution. The dashed vertical lines within each plot indicate the specific reference values. Pre, presurgery; $6 \mathrm{M}, 12 \mathrm{M}$, and $24 \mathrm{M}$, respectively, 6, 12, and 24 months after surgery.
In the cholesterol-HDL ratio, a major decline was seen after 6 months in both groups, similar to the total cholesterol concentration (Fig. 2c). However, where cholesterol concentrations increased during the follow-up, the cholesterol-HDL ratio further decreased. Also, the shape of the beanplot changed over time, having smaller distribution widths. The largest decline was observed in the RYGB group after 24 months, with a median cholesterol-HDL ratio of $2.7(2.3-3.2)$ compared to $3.1(2.6-3.8)$ in the SG group $(p<0.001$; Table 2$)$.

Thus, the improvement in the lipid profile was larger in RYGB patients compared to SG patients. Although the degree of decrease in triglyceride concentrations was equal in both groups, the improvement in cholesterol concentrations and the cholesterol-HDL ratio was larger in the RYGB group. This shows that RYGB results in a larger decrease in lipid profiles compared to SG.

\section{Liver Enzymes (AST, ALT, and ALP)}

Presurgically, the enzymatic activity of AST was higher in SG patients. With both types of surgery a decline was seen 6 months after surgery. During further follow-up, activities stabilized in patients who had undergone SG; however, after RYGB the AST activity gradually increased toward the preoperative level (Fig. 3a). After 2 years, this resulted in a small, statistically significantly lower AST activity in SG patients, i.e., 22 (19-26) U/L compared to 20 (17-24) U/L $(p<0.001$; Table 2).

Similar to AST, the ALT activity before surgery was higher in SG patients compared to RYGB patients. Six months after surgery, a major decrease was seen in both groups (Fig. 3b). However, this decline was larger in the SG group. Thereafter, the median ALT activity stabilized in both groups. Two years after surgery, ALT activity was significantly lower in the SG group, i.e., 22 (17-30) U/L compared to 19 (15-23) U/L $(p<0.001$; Table 2). The width of the distribution of ALT activities decreased after surgery for both surgery types, and the proportion of patients within the reference range increased after surgery (Fig. 3b).

The ALP activity in patients who had undergone RYGB was found to be minimally increased at the 6 months follow-up, after which the ALP activity stabilized (Fig. 3c). In the SG group a gradual decline was seen, resulting in a significantly lower ALP activity after SG, i.e., $86(72-101)$ IU/L compared to 72 (61-86) IU/L $(p<$ 0.001 ; Table 2). The beanplot shows a normally distributed ALP activity in both groups. Both groups started equally before surgery but shifted in opposite directions during follow-up (Fig. 3c).

Thus, the activity of liver enzymes before surgery was equal or higher in SG patients compared to RYGB patients. However, after surgery the enzyme activity decreased significantly more in SG patients compared to RYGB patients. So, after surgery the situation was the other way around, and SG patients had a lower activity of liver enzymes compared to RYGB patients. The combination of both effects seems to reveal that SG results in a larger decrease of liver enzyme activity compared to RYGB. 


\section{Calcium Regulation (PTH and Calcium)}

Calcium concentrations slowly decreased during follow-up in both groups, particularly in RYGB patients, while albumin concentrations remained constant in both groups, i.e., $44(42-45) \mathrm{g} / \mathrm{L}(p=0.92)$. However, the difference in median calcium levels 24 months after surgery was relatively small, i.e., $2.35(2.29-2.40) \mathrm{mmol} / \mathrm{L}$ in the SG group compared to $2.32(2.27-2.38) \mathrm{mmol} / \mathrm{L}$ in the RYGB group ( $p<0.001$; Table 2). The observed distributions of calcium concentration were normal in both groups, and the majority of the population remained within the reference interval (Fig. 4a). The proportion outside the reference interval was larger on the righthand side (hypercalcemia) compared to the left-hand side (hypocalcemia).

In both SG and, to a lesser extent, RYGB patients, an immediate decrease in PTH concentrations was seen 6 months after surgery, progressing slowly to an increase in further follow-up (Fig. 4b). Before surgery, SG patients had a higher median PTH concentration compared to RYGB patients (respectively, 6.8 [5.2-8.7] and 6.4 [5.08.2] pmol/L; $p<0.01$; Table 2). However, 6 months after surgery, SG patients showed a larger decrease in PTH concentrations, with a median of $4.9(3.8-6.4)$ versus 5.2 (4.1-6.8) $\mathrm{pmol} / \mathrm{L}$ in RYGB patients $(p<0.01)$. Twentyfour months after surgery, PTH concentrations had increased in both groups but a lower PTH concentration was seen in patients after SG compared to RYGB (respectively, 5.4 [4.3-6.9] and 5.9 [4.6-7.4] pmol/L; $p<0.001$ ). The beanplot showed that before surgery significantly larger proportions of both populations had PTH values above the upper reference limit compared to after surgery (Fig. 4b).

\section{Discussion}

Rapid progress has been made in clinical analytics, i.e., techniques for analyzing large quantities of data and gleaning new insights from that analysis, which is part of "big data." As a result, there are new opportunities to use big data and data analytics to hit the target of the Triple Aim goals, i.e., to improve the health of the population, improve the patient experience, and lower healthcare costs. With smart analysis tools, one can easily analyze large data sets and compare different patient groups. As a result, research can be performed at the population level instead of in a trial setting, often limited by a selective scope or a low number of included patients. After identifying patterns in the data, one wants to understand why the patterns are as they appear and link them with medical knowledge.

In this study, the opportunity has been seized to apply smart visualization on a large set of laboratory data, unraveling patterns that even go against the current literature. In this study the somewhat uncommon modality of beanplots was used. The advantage over traditional graphics is an easier insight into differences between SG and RYGB, just at a glance. In addition, it provides an easy overview of the distribution over time and of the complete population, as skewness and kurtosis are also shown. Moreover, in the context of corresponding references or target values, one can directly estimate the proportion of the population within or outside the reference range. Beanplots also show whether there is a continuous distribution of observed values or multiple populations with overlapping distributions. In our opinion, this type of visualization is more accessible and thus provides more insight for the clinician into the information than mere representation of the mean, median, SD, IQR, and/or box plots.

\section{Glucose Metabolism (Hb1Ac)}

In both types of surgery, a significant, stable decrease in HbA1c levels was seen after 6 months. Comparable results were found in a recent meta-analysis, i.e., no superior glycemic control for one technique in the short or long term (3-5 years of follow-up). The authors revealed that baseline BMI, preoperative fasting plasma glucose, the duration of T2DM, and preoperative $\mathrm{Hb} 1 \mathrm{Ac}$ had a predictive value for glycemic control after SG, but only the latter 2 correlated with glycemic control after RYGB. However, results from RCT on this subject have been inconsistent [17-19]. The regulation of glucose and lipid metabolism by the gastrointestinal tract is not yet well understood.

\section{Cholesterol (Triglycerides, Total Cholesterol, and the} Cholesterol-HDL Ratio)

Our study showed a reduction in triglycerides, total cholesterol, and the cholesterol-HDL ratio after RYGB and SG. After 2 years of surgery, concentrations of triglycerides, total cholesterol, and the cholesterol-HDL ratio were found to be significantly lower after RYGB than after SG. These findings are in line with other studies [2024]. The superior efficacy of RYGB compared to the restrictive technique of SG has mainly been attributed to the malabsorptive nature of RYGB, as this leads to a decreased cholesterol absorption, possibly as a result of reduced sterol absorption in conjunction with enhanced hepatic ste-
28

Obes Facts 2021;14:21-31 DOI: $10.1159 / 000510401$ van Berckel et al. 
rol catabolism as reflected mainly by bile acid synthesis $[24,25]$. These effects are surgery-specific and independent of weight loss or any improvement of glucose metabolism [24, 25].

\section{Liver Enzymes (AST, ALT, and ALP)}

Obesity is associated with nonalcoholic fatty liver disease, reflected in histologic changes in liver parenchyma as well as liver function alterations [24]. Bariatric surgery is expected to halt and reverse liver damage mainly by inducing weight loss $[26,27]$. Our results showed improvement of liver function in normalization of ALT in both SG and RYGB. In comparison with RYGB, we found significant lower activities of AST, ALT, and ALP in patients 2 years after SG. This is in line with a recent prospective study with 1,037 patients [28] and other studies [26, 2931]. The rapid improvement of, especially, ALT within the first 6 months after SG has been reported before [27]. The mechanism behind these fast improvements in ALT after SG is unclear. It has been postulated that patients with liver abnormalities in general may recover more easily after weight loss than those with other obesity-related disorders, as previous studies have demonstrated rapid changes in liver size, reflective of steatosis, after just 2 weeks of very-low-calorie diets [32]. Others believe that normalization of the liver function might play a role in resolution of metabolic disease [33]. Another possible reason for the transient deterioration and/or late recovery of liver function after RYGB might be that malabsorption may aggravate the suboptimal reparative capacity and antioxidant reserve of the liver in a setting of increased free fatty acid mobilization, oxidation, and free radical species production caused by rapid weight loss [34-36]. Experimental evidence suggest that SG may act through changes in bile acid metabolism and the related changes in signaling through the farsenoid $\mathrm{X}$ receptor, which affects the fatty acid metabolism of the liver. However, whether these mechanisms are responsible for the effects of SG in patients remains elusive and requires further research $[34,37,38]$.

\section{Calcium Regulation (PTH and Calcium)}

We found lower calcium levels and higher PTH levels in patients 2 years after RYGB in comparison to SG. A recent meta-analysis confirmed our results regarding the difference in calcium and PTH between RYGB and LG [39]. Another recent meta-analysis showed a higher PTH level but similar calcium levels between the 2 groups [40]. Considerable heterogeneity was detected in this metaanalysis. The malabsorptive component of RYGB (the calcium absorption site in the duodenum is bypassed) instead of the restrictive component of SG could explain the difference in calcium and subsequent PTH. Although PTH is mainly regulated by calcium, no information regarding phosphorus and vitamin $\mathrm{D}$ is available, failing an explanation of the interesting drop after 6 months and the subsequent rise of PTH after RYGB. More research is needed on this subject.

This study has some limitations. In this retrospective study, preexisting data were analyzed so bias in the selection of patients is not excluded. Patients were only included for visual analysis when there was data available for each selected time point, i.e., before surgery and 6,12, and 24 months after surgery. This criterion was set to allow for analysis of the same patient population over time, a so called "complete case" analysis. The influence of this selection on the presented results was assumed to be minor as the observed differences in baseline patient characteristics were relatively small (data not shown). The reason for missing one or more follow-ups by the excluded patients was assumed to be random. One may hypothesize that only well-performing patients return to their appointments and have blood tests performed, so the effects presented in this study may reflect an overestimation of the actual effect of bariatric surgery. Even though ours was a large cohort with only primary bariatric surgeries, we believe that a longer follow-up period could provide additional important information. Another limitation is the significant difference in baseline age, BMI, and hypertension, although I was relatively small. Unfortunately, additional data on the reasoning behind the choice of either SG or RYGB were not available. An additional major drawback is the lack of information regarding the medication used, the use of supplements, the medical history, or more information regarding nonalcoholic fatty liver disease (i.e., liver biopsies).

In conclusion, beanplots allow intuitive visualization of population distributions. Though retrospective, the analysis of this large population-based bariatric data set using beanplots suggests a comparable efficacy in reducing diabetes with both types of surgery. RYGB surgery reduced dyslipidemia more effectively than $S G$. The trend toward a more pronounced decrease in liver enzyme activities following SG is a subject for further investigation.

\section{Acknowledgement}

We thank Ruben Deneer, MSc, for his input during the initial data analysis and data visualization. 


\section{Statement of Ethics}

The local medical ethical board waived ethical approval for this study. Formal consent is not required for this type of study. However, all of the patients signed an informed consent form for the use of their anonymized data for research purposes.

\section{Conflict of Interest Statement}

The authors declare no conflict of interests.

\section{Funding Sources}

There were no sources of support for this study.

\section{Author Contributions}

M.M.G.B. and S.L.M.L.: data acquisition, statistical analysis, and drafting of this paper. A.-K.B., V.S., and S.W.N.: critical revision of this paper. All of the authors were involved in the writing of this paper and gave final approval for the submitted and published versions.

\section{References}

1 Buchwald H, Avidor Y, Braunwald E, Jensen MD, Pories W, Fahrbach K, et al. Bariatric surgery: a systematic review and meta-analysis. JAMA. 2004 Oct;292(14):1724-37.

2 Maggard MA, Shugarman LR, Suttorp M, Maglione M, Sugerman HJ, Livingston EH, et al. Meta-analysis: surgical treatment of obesity. Ann Intern Med. 2005 Apr;142(7):54759.

3 Christou NV, Sampalis JS, Liberman M, Look D, Auger S, McLean AP, et al. Surgery decreases long-term mortality, morbidity, and health care use in morbidly obese patients. Ann Surg. 2004 Sep;240(3):416-23.

4 Sjöström L, Narbro K, Sjöström CD, Karason K, Larsson B, Wedel H, et al.; Swedish Obese Subjects Study. Effects of bariatric surgery on mortality in Swedish obese subjects. N Engl J Med. 2007 Aug;357(8):741-52.

5 Parrott J, Frank L, Rabena R, Craggs-Dino L, Isom KA, Greiman L. American Society for Metabolic and Bariatric Surgery Integrated Health Nutritional Guidelines for the Surgical Weight Loss Patient 2016 Update: micronutrients. Surg Obes Relat Dis. 2017 May;13(5): $727-41$.

6 Mechanick JI, Youdim A, Jones DB, Timothy Garvey W, Hurley DL, Molly McMahon M, et al. Clinical practice guidelines for the perioperative nutritional, metabolic, and nonsurgical support of the bariatric surgery patient 2013 update: cosponsored by American Association of Clinical Endocrinologists, the Obesity Society, and American Society for Metabolic \& Bariatric Surgery. Surg Obes Relat Dis. 2013 Mar-Apr;9(2):159-91.

7 Fried M, Yumuk V, Oppert JM, Scopinaro N, Torres A, Weiner R, et al.; International Federation for Surgery of Obesity and Metabolic Disorders-European Chapter (IFSO-EC); European Association for the Study of Obesity (EASO); European Association for the Study of Obesity Obesity Management Task Force (EASO OMTF). Interdisciplinary European guidelines on metabolic and bariatric surgery. Obes Surg. 2014 Jan;24(1):42-55.
8 le Roux CW, Welbourn R, Werling M, Osborne A, Kokkinos A, Laurenius A, et al. Gut hormones as mediators of appetite and weight loss after Roux-en-Y gastric bypass. Ann Surg. 2007 Nov;246(5):780-5.

9 Dimitriadis E, Daskalakis M, Kampa M, Peppe A, Papadakis JA, Melissas J. Alterations in gut hormones after laparoscopic sleeve gastrectomy: a prospective clinical and laboratory investigational study. Ann Surg. 2013 Apr;257(4):647-54.

10 Ramos A, Kow L, Brown W, Welbourn R, Dixon J, Kinsman R, Walton P. Fifth IFSO Global Registry Report 2019. Reading: Dendrite Clinical Systems; 2019.

11 Kwon Y, Kim HJ, Lo Menzo E, Park S, Szomstein S, Rosenthal RJ. Anemia, iron and vitamin B12 deficiencies after sleeve gastrectomy compared to Roux-en-Y gastric bypass: a meta-analysis. Surg Obes Relat Dis. 2014 JulAug;10(4):589-97.

12 Kampstra P. Beanplot: A Boxplot Alternative for Visual Comparison of Distributions. J Stat Softw. 2008;28(1):1-9.

13 Muthers S, Matzarakis A. Use of beanplots in applied climatology: a comparison with boxplots. Meteorol Z. 2010;19(6):641-44.

14 Drago C, Lauro C, Scepi G. Beanplot Data Analysis in a Temporal Framework. In: Giudici P, Ingrassia S, Vichi M, editors. Statistical Models for Data Analysis. Studies in Classification, Data Analysis, and Knowledge Organization. Heidelberg: Springer; 2013. https:// doi.org/10.1007/978-3-319-00032-9_14.

15 Drago C, Scepi G. Visualizing and exploring high frequency financial data: Beanplot time series. In: Ingrassia S, Rocci R, Vichi M, editors. New perspectives in statistical modeling and data analysis: Studies in classification, data analysis, and knowledge organization. Berlin: Springer; 2011.

16 Brethauer SA, Kim J, el Chaar M, Papasavas P, Eisenberg D, Rogers A, et al.; ASMBS Clinical Issues Committee. Standardized outcomes reporting in metabolic and bariatric surgery. Surg Obes Relat Dis. 2015 May-Jun; 11(3):489-506.
17 Schauer PR, Bhatt DL, Kirwan JP, Wolski K, Aminian A, Brethauer SA, et al.; STAMPEDE Investigators. Bariatric Surgery versus Intensive Medical Therapy for Diabetes - 5-Year Outcomes. N Engl J Med. 2017 Feb;376(7): 641-51.

18 Lee WJ, Chong K, Ser KH, Lee YC, Chen SC, Chen JC, et al. Gastric bypass vs sleeve gastrectomy for type 2 diabetes mellitus: a randomized controlled trial. Arch Surg. 2011 Feb;146(2):143-8.

19 Keidar A, Hershkop KJ, Marko L, Schweiger C, Hecht L, Bartov N, et al. Roux-en-Y gastric bypass vs sleeve gastrectomy for obese patients with type 2 diabetes: a randomised trial. Diabetologia. 2013 Sep;56(9):1914-8.

20 Vix M, Diana M, Liu KH, D’Urso A, Mutter $\mathrm{D}, \mathrm{Wu} \mathrm{HS}$, et al. Evolution of glycolipid profile after sleeve gastrectomy vs. Roux-en-Y gastric bypass: results of a prospective randomized clinical trial. Obes Surg. 2013 May; 23(5):613-21.

21 Griffo E, Cotugno M, Nosso G, Saldalamacchia G, Mangione A, Angrisani L, et al. Effects of Sleeve Gastrectomy and Gastric Bypass on Postprandial Lipid Profile in Obese Type 2 Diabetic Patients: a 2-Year Follow-up. Obes Surg. 2016 Jun;26(6):1247-53.

22 Cunha FM, Oliveira J, Preto J, Saavedra A, Costa MM, Magalhães $\mathrm{D}$, et al. The Effect of Bariatric Surgery Type on Lipid Profile: An Age, Sex, Body Mass Index and Excess Weight Loss Matched Study. Obes Surg. 2016 May; 26(5):1041-7.

23 Benaiges D, Flores-Le-Roux JA, Pedro-Botet J, Ramon JM, Parri A, Villatoro M, et al.; Obemar Group. Impact of restrictive (sleeve gastrectomy) vs hybrid bariatric surgery (Rouxen-Y gastric bypass) on lipid profile. Obes Surg. 2012 Aug;22(8):1268-75.

24 Benetti A, Del Puppo M, Crosignani A, Veronelli A, Masci E, Frigè F, et al. Cholesterol metabolism after bariatric surgery in grade 3 obesity: differences between malabsorptive and restrictive procedures. Diabetes Care. 2013 Jun;36(6):1443-7. 
25 Pihlajamäki J, Grönlund S, Simonen $\mathrm{M}$, Käkelä P, Moilanen L, Pääkkönen $\mathrm{M}$, et al. Cholesterol absorption decreases after Rouxen-Y gastric bypass but not after gastric banding. Metabolism. 2010 Jun;59(6):866-72.

26 Burza MA, Romeo S, Kotronen A, Svensson PA, Sjöholm K, Torgerson JS, et al. Long-term effect of bariatric surgery on liver enzymes in the Swedish Obese Subjects (SOS) study. PLoS One. 2013;8(3):e60495.

27 Ooi GJ, Burton PR, Doyle L, Wentworth JM, Bhathal PS, Sikaris K, et al. Effects of Bariatric Surgery on Liver Function Tests in Patients with Nonalcoholic Fatty Liver Disease. Obes Surg. 2017 Jun;27(6):1533-42.

28 Motamedi MA, Khalaj A, Mahdavi M, Valizadeh M, Hosseinpanah F, Barzin M. Longitudinal Comparison of the Effect of Gastric Bypass to Sleeve Gastrectomy on Liver Function in a Bariatric Cohort: Tehran Obesity Treatment Study (TOTS). Obes Surg. 2019 Feb; 29(2):511-8.

29 Billeter AT, Senft J, Gotthardt D, Knefeli P, Nickel F, Schulte T, et al. Combined Non-alcoholic Fatty Liver Disease and Type 2 Diabetes Mellitus: Sleeve Gastrectomy or Gastric Bypass?-a Controlled Matched Pair Study of 34 Patients. Obes Surg. 2016 Aug;26(8):186774.
30 Bower G, Toma T, Harling L, Jiao LR, Efthimiou E, Darzi A, et al. Bariatric Surgery and Non-Alcoholic Fatty Liver Disease: a Systematic Review of Liver Biochemistry and Histology. Obes Surg. 2015 Dec;25(12):2280-9.

31 Kalinowski P, Paluszkiewicz R, ZiarkiewiczWróblewska B, Wróblewski T, Remiszewski $\mathrm{P}$, Grodzicki M, et al. Liver Function in $\mathrm{Pa}$ tients With Nonalcoholic Fatty Liver Disease Randomized to Roux-en-Y Gastric Bypass Versus Sleeve Gastrectomy: A Secondary Analysis of a Randomized Clinical Trial. Ann Surg. 2017 Nov;266(5):738-45.

32 Colles SL, Dixon JB, Marks P, Strauss BJ, O'Brien PE. Preoperative weight loss with a very-low-energy diet: quantitation of changes in liver and abdominal fat by serial imaging. Am J Clin Nutr. 2006 Aug;84(2):304-11.

33 Taylor R. Pathogenesis of type 2 diabetes: tracing the reverse route from cure to cause. Diabetologia. 2008 Oct;51(10):1781-9.

34 van Zutphen T, Ciapaite J, Bloks VW, Ackereley C, Gerding A, Jurdzinski A, et al. Malnutrition-associated liver steatosis and ATP depletion is caused by peroxisomal and mitochondrial dysfunction. J Hepatol. 2016 Dec; 65(6):1198-208.
35 Csak T, Ganz M, Pespisa J, Kodys K, Dolganiuc A, Szabo G. Fatty acid and endotoxin activate inflammasomes in mouse hepatocytes that release danger signals to stimulate immune cells. Hepatology. 2011 Jul;54(1):13344.

36 Verna EC, Berk PD. Role of fatty acids in the pathogenesis of obesity and fatty liver: impact of bariatric surgery. Semin Liver Dis. 2008 Nov;28(4):407-26

37 Myronovych A, Kirby M, Ryan KK, Zhang W, Jha P, Setchell KD, et al. Vertical sleeve gastrectomy reduces hepatic steatosis while increasing serum bile acids in a weight-loss-independent manner. Obesity (Silver Spring). 2014 Feb;22(2):390-400

38 Boden G. Effects of free fatty acids (FFA) on glucose metabolism: significance for insulin resistance and type 2 diabetes. Exp Clin Endocrinol Diabetes. 2003 May;111(3):121-4.

39 Tian Z, Fan XT, Li SZ, Zhai T, Dong J. Changes in Bone Metabolism After Sleeve Gastrectomy Versus Gastric Bypass: a Meta-Analysis. Obes Surg. 2020 Jan;30(1):77-86.

40 Gu L, Fu R, Chen P, Du N, Chen S, Mao D, et al. In Terms of Nutrition, the Most Suitable Method for Bariatric Surgery: Laparoscopic Sleeve Gastrectomy or Roux-en-Y Gastric Bypass? A Systematic Review and Meta-analysis. Obes Surg. 2020 May;30(5):2003-14. 